\title{
Éditorial LUCA, énigmatique cellule mère universelle प.
}

> Les premières cellules vivantes apparaissent sans doute sur Terre il y a 3,5 milliards d'années, la fourchette d'incertitude restant grande: de plus de 3,8 à 3,2 milliards d'années. Les cellules originelles, comme leurs équivalents modernes, devaient être caractérisées par une membrane séparant un milieu intérieur d'un milieu extérieur; une capacité de croissance par métabolisme de molécules extérieures, ce qui implique l'utilisation d'une source d'énergie; une capacité pour la cellule de se diviser, et donc de proliférer; et, enfin, un programme génétique assurant une certaine stabilité des propriétés biologiques de la cellule et leur transmission aux cellules filles. Une autre caractéristique de ce programme génétique des origines est de n'être pas recopié exactement à l'identique lors de la division des cellules. II s'ensuit une diversité biologique, accrue par les transferts horizontaux de matériel génétique, qui constitue le substratum de l'évolution des espèces. Ceux des descendants d'un organisme parental ayant, par hasard, hérité de modifications ou, par transfert, de combinaisons de caractères nuisant à leur reproduction dans un écosystème donné en sont progressivement éliminés. À l'inverse, les êtres dotés de traits favorisant leur prolificité ont une descendance nombreuse, ce qui assure la large diffusion, et donc le succès des gènes et combinaisons de gènes conférant un tel avantage sélectif.

C'est de ces premières protocellules qu'émergeront toutes les formes de vie que l'on connaît aujourd'hui. Depuis les travaux de Carl Woese, un bactériologiste de l'Illinois, à la fin des années 60 , on distingue trois embranchements fondamentaux des formes de vie, les bactéries (ou eubactéries), les archaeas (archaeabactéries) et les eucaryotes [1]. Ces derniers sont pourvus d'un noyau, ce qui n'est pas le cas des bactéries et des archaeas qui sont des procaryotes. Initialement, l'observation selon laquelle certaines familles d'archaeas se trouvent vivre dans des conditions extrêmes de température, de salinité ou (et) de $\mathrm{pH}$, suggéra qu'elles représentaient le rameau initial dont ont divergé les bactéries et les eucaryotes. Carl Woese avait fondé ses premiers travaux sur la comparaison de la séquence des ARN ribosomiques des êtres vivants, ce qui ne donnait strictement aucune indication quant à l'arbre évolutif qui a présidé à l'émergence de ces types de cellules. L'analyse génétique et biochimique d'un plus grand nombre de bactéries, d'archaeas et d'eucaryotes allait cependant jeter un sérieux doute sur la précession des archaeas.

En effet, par certains côtés, les eucaryotes ressemblent plus aux archaeas qu'aux bactéries. Tel est le cas des enzymes de la machinerie de réplication et de transcription. De plus, I'ADN des archaeas forme des complexes avec des assemblées protéiques évoquant les nucléosomes eucaryotiques. En revanche, eucaryotes et bactéries présentent plus de similarités quant aux enzymes gouvernant le métabolisme. Globalement, les gènes codant les fonctions nucléaires ont tendance à évoquer plutôt les archaeas alors que ceux codant les étapes du métabolisme ont un type plutôt bactérien. Ces observations ont conduit à formuler l'hypothèse selon laquelle les eucaryotes dériveraient d'une symbiose datant d'il y a environ 2,6 à 2,8 milliards d'années entre une $\alpha$-protéobactérie et une archaea méthanogène, expliquant à la fois l'origine double du génome eucaryotique et l'apparition des mitochondries $[2,3]$. Selon ce schéma, la cellule originelle serait bactérienne; elle aurait divergé pour donner les archaeas puis, après fusion de descendants des deux lignages dérivant de cet embranchement, les eucaryotes, d'emblée dotés de mitochondries qui auraient par la suite été perdues dans de rares rameaux.

LUCA (Last Universal Common Ancester), le dernier ancêtre commun à toutes les cellules vivantes, serait par conséquent une bactérie. Les travaux de phylogénie moléculaire ne sont pas incompatibles avec ce schéma mais ne permettent pas de déterminer, dans l'hypothèse où les cellules bactériennes auraient précédé toutes les autres, à quelles familles vivant à l'heure actuelle elles pouvaient ressembler le plus. Pour certains, il pourrait s'agir de bactéries thermophiles de type Aquificales ou Thermotogales. Cependant, il n'est pas aisé de comprendre comment, aux origines de la vie, des enzymes thermostables auraient déjà pu exister. De fait, d'autres groupes privilégient l'hypothèse selon laquelle notre mère supérieure serait une bactérie vivant à température modérée, ressemblant par exemple aux Planctomycetales dont la membrane, comme celle des archaeas, est dépourvue de peptidoglycane et dont les chromosomes sont entourés d'une membrane, ce qui évoque bien entendu le possible ancêtre d'un noyau $[4,5]$.

Patrick Forterre favorise quant à lui une hypothèse révolutionnaire selon laquelle, après tout, ce serait les eucaryotes, les cellules dont nous sommes constitués, qui constitueraient l'ancêtre universel de la vie moderne $[4,5]$. Par certains aspects, observe Patrick Forterre, les bactéries semblent s'être modernisées par rapport aux eucaryotes, voire aux archaeas. Les eucaryotes ont des chromosomes linéaires, ce qui exige la mise en œuvre d'une procédure compliquée de réparation permanente des extrémités via la constitution des télomères. Les nombreux introns des 
eubactéries, dont quelques exemples de structures différentes existent chez les archaeas, doivent être excisés des transcrits ARN par une machinerie compliquée, celle des splicéosomes. L'ARN ne peut être traduit immédiatement après sa transcription, il doit subir la maturation d'excision-épissage, puis être transporté vers le cytoplasme avant que de servir de matrice pour la synthèse protéique. Les eubactéries ont gagné beaucoup d'efficacité en tous ces domaines. Leur chromosome circulaire règle le problème de la réparation des extrémités. L'absence d'intron et de membrane nucléaire permet à la traduction protéique de débuter avant même que la transcription ne soit achevée, ce qui accroît dans de grandes proportions la capacité de ces cellules à synthétiser des protéines et explique peut-être leur très remarquable succès évolutif. $\mathrm{Nul}$ être ne semble avoir en effet autant de capacités adaptatives que les eubactéries qui, sans doute, persisteraient même si des catastrophes écologiques ou nucléaires faisaient disparaître toute autre forme de vie sur terre.

On peut donc faire l'hypothèse selon laquelle une protocellule ancestrale, sac de matières organiques, aurait été infectée par un virus dont l'existence dans la soupe primitive prébiotique pourrait avoir précédé l'apparition des premières cellules $[6,7]$. De fait, les différents scénarios d'origine de la vie supposent que des molécules réplicatives existaient avant la formation des premières cellules. Or, c'est bien là une caractéristique d'un virus, petit complexe acellulaire composé d'un génome et de quelques protéines facilitant le parasitage d'une cellule. La perte ultérieure du noyau et différents perfectionnements de nature à accroître l'efficacité biosynthétique des micro-organismes seraient à l'origine des archaeas, puis des bactéries.

La seule piste ouverte pour déterminer ce que pouvaient êtres les caractéristiques de LUCA est la recherche de gènes conservés chez tous les êtres vivants modernes, archaeas, bactéries et eucaryotes. Le séquençage de plus de quatre cents génomes complets donne le moyen d'une telle étude $[4,5,8]$. Elle est décevante: on ne note que soixante à quatre-vingt «protéines universelles » candidates à représenter les reliques de l'équipement de LUCA. Bien entendu, la dernière cellule commune aux embranchements actuels de la vie ne pouvait qu'être plus complexe. II manque donc de l'information. De plus, les facteurs et protéines intervenant dans la traduction protéique sont surreprésentés ( $80 \%$ ) et les enzymes gouvernant la réplication, la réparation et la recombinaison de l'ADN manquent presque totalement. Qu'est-ce à dire?

Peut-être, après tout, LUCA n'existait-t-il pas. Telle est l'hypothèse défendue par Carl Woese qui pense qu'avant la divergence des différents embranchements, une communauté cellulaire possédant des génomes à ARN échangeait librement du matériel biologique, comme le font encore en une large mesure, aujourd'hui, les bactéries $[8,9]$. De nouvelles propriétés, privilégiant la stabilité au sein du lignage et diminuant les échanges, seraient apparues chez des membres particuliers de cette collectivité, permettant ainsi de sélectionner certains ensembles distincts et avantageux de caractères en les protégeant d'une re-homogénéisation permanente.
Une autre hypothèse, privilégiée par Patrick Forterre et d'autres scientifiques, est que le dernier ancêtre commun universel a bel et bien existé, mais était un organisme à ARN. L'ADN I'aurait remplacé suite à des événements indépendants ultérieurs survenant dans des embranchements séparés $[4,5]$. L'apport d'information d'origine virale pourrait, là aussi, expliquer cette évolution convergente. Cependant, cette hypothèse virale, bien commode, a l'inconvénient de convenir sans distinction aux différents schémas proposés, et donc d'être peu discriminative. Un LUCA à ADN aurait aussi bien pu engendrer des branches au niveau desquelles la machinerie de réplication - réparation - recombinaison de l'ADN (les $3 R$ ) aurait, à différentes périodes, été remplacée par des apports viraux.

Au total, la messe n'est pas dite, et sans doute ne le sera-t-elle jamais de façon définitive. La nature de notre mère cellulaire supérieure à tous continuera de constituer un champ fécond pour l'imagination et l'esprit de synthèse des biologistes du futur. L'auteur de cet éditorial désirant participer à cette émulation se doit d'afficher ses préférences, en les présentant comme telles et non comme des probabilités. Si les premières cellules étaient de type bactérien, la généralité des échanges de matériel entre elles proposée par Carl Woese semble vraisemblable. De plus, la précession des fulgurances évolutives - les lignages distincts - par une période collectiviste où, au gré des réassortiments permanents et aléatoires de molécules codantes, tous les assemblages peuvent être testés, m'apparaît dotée d'un bien plus grand potentiel évolutif que le seul processus mutationnel au sein de microorganismes dépourvus de sexualité. La multiplication des combinaisons possibles a pu accroître les chances que certaines d'entre elles soient de qualité telle qu'il fut avantageux pour l'évolution d'en préserver les caractéristiques, eubactériennes, archéales ou eucaryotiques. Plusieurs milliards d'années après, c'est l'intérêt retrouvé de la diversification génétique par échange d'ADN qui expliquera un autre grand succès évolutif, celui de la sexualité. $\diamond$

LUCA, the enigmatic universal cell mother

A. Kahn

Institut Cochin, IFR Alfred Jost, 22, rue Méchain, 75014 Paris, France.

kahn@cochin.inserm.fr

\section{RéFÉRENCES}

1. Woese CR, Fox GE. The concept or cellular evolution. J Mol Evol 1977 ; 10 : 1-6.

2. Horiike T, Hamada K. Kanaya S, Shinozawa T. Origin of eukaryotic cell nuclei by symbiosis of Archaea in Bacteria is revealed by homology-hit analysis. Nat Cell Biol $2001 ; 3: 210-4$.

3. Martin W, Embley TM. Early evolution comes full circle. Nature 2004 ; 431 : 134-7.

4. Hamilto G. Mother superior. New Scientist 2005 ; (3 septembre) : 26-9.

5. Forterre P. LUCA : à la recherché du plus proche ancêtre commun universel. Med Sci (Paris) $2005 ; 21: 860-5$.

6. Balter M. Evolution on life's fringes. Science $2000 ; 289$ : 1866-7.

7. Pennisi $\varepsilon$. The birth of the nucleus. Science $2004 ; 305: 766-8$.

8. Whitfield J. Born in a watery commune. Nature $2004 ; 427: 674-6$.

9. Woese CR. On the evolution of cells. Proc Natl Acad Sci USA $2002 ; 99: 8742-7$.

\section{TIRÉS À PART}

A. Kahn 